\title{
Metastatic and non-metastatic sentinel inguinofemoral lymph nodes in vulvar cancer show an increased lymphangiogenesis
}

\author{
Magda Piechowicz ${ }^{1, A-B, D-F} \oplus$, Marcin Mikos ${ }^{2, C, E-F} \oplus$, Tomasz Banas ${ }^{1, C-F} \oplus$, Krzysztof Okon $^{3, B, E-F} \oplus$, \\ Milosz Pietrus ${ }^{1, D-F}{ }^{\oplus}$, Marta Balajewicz-Nowak ${ }^{1, B, E-F} \oplus$, Lukasz Szczudlik ${ }^{1, C, E-F} \oplus$, Zbigniew Kojs ${ }^{4, C, E-F} \oplus$, \\ Aleksandra Czerw ${ }^{5,6, C, E-F \oplus}$, Grzegorz Juszczyk ${ }^{7,5, C, E-F \oplus}$, Kazimierz Pityński ${ }^{1, A-F} \oplus$ \\ ${ }^{1}$ Department of Gynecology and Oncology, Jagiellonian University Medical College, Krakow, Poland \\ ${ }^{2}$ Faculty of Medicine and Health and Medical Science, Andrzej Frycz Modrzewski University, Krakow Poland \\ ${ }^{3}$ Department of Pathomorphology, Jagiellonian University Medical College, Krakow, Poland \\ ${ }^{4}$ Oncology Centre - M. Skłodowska-Curie Institute, Krakow, Poland \\ ${ }^{5}$ Department of Economic and System Analyses, National Institute of Public Health - National Institute of Hygiene, \\ Warsaw, Poland \\ ${ }^{6}$ Department of Health Economics and Medical Law, Medical University of Warsaw, Warsaw, Poland \\ ${ }^{7}$ Department of Public Health, Faculty of Health Science, Medical University of Warsaw, Warsaw, Poland \\ A - Research concept and design, B - Collection and/or assembly of data, C - Data analysis and interpretation, \\ $D$ - Writing the article, E - Critical revision of the article, F - Final approval of article
} Piechowicz M, Mikos M, Banas T, Okon K, Pietrus M, Balajewicz-Nowak M, Szczudlik L, Kojs Z, Czerw A, Juszczyk G, Pityński K. Metastatic
and non-metastatic sentinel inguinofemoral lymph nodes in vulvar cancer show an increased lymphangiogenesis. Ann Agric Environ Med. 2020; 27(1): 123-128. doi: 10.26444/aaem/105925

\section{Abstract}

Introduction and objective. Lymph node involvement is a strong predictor of disease recurrence and patient survival in vulvar cancer. The aim of the study was to evaluate the feasibility of sentinel lymph node (SLN) screening, the incidence of skip metastases, and lymph node lymphangiogenesis.

Materials and method. Fifty-five patients participated in this prospective, single centre study. A double SLN screening method was employed using radiocolloid (technetium-99 sulfur colloid) and $1.0 \%$ Isosulfan Blue. Immunohistochemistry, using a mouse monoclonal antibody against D2-40, was used to evaluate lymphatic vessel density (LVD). All calculations were performed using STATISTICA software v. 10 (StatSoft, USA, 2011); $p<0.05$ was considered significant.

Results. Using both methods of SLN detection, $100 \%$ accuracy was achieved, and skip metastases were diagnosed in only one woman (1.82\%). Peri-tumour median LVD was significantly increased compared with matched intra-tumour samples $(p<0.001)$, while median LVD was significantly lower in negative, compared with positive SLN, regardless of whether matched non-SLN were negative $(p<0.001)$ or positive $(p=0.005)$. Metastatic SLN exhibited significantly higher median LVD compared with matched negative non-SLN $(p=0.015)$, while no significant difference in median LVD was detected between positive SLN and matched positive non-SLN. However, negative SLN had a significantly higher median LVD compared with matched negative non-SLN ( $p=0.012)$.

Conclusions. SLN detection is a safe and feasible procedure in vulvar cancer. In patients without nodular involvement, SLN, compared with non-SLN, exhibited significantly higher median LVD, which may be an indication of its preparation to host metastases, and thus requires further investigation.

\section{Key words}

anti-D2-40 antibody; lymphatic vessel density; immunohistochemistry; sentinel lymph node; vulvar cancer

\section{INTRODUCTION}

Vulvar carcinomas, among which the squamous type is most frequent, are rare gynaecological malignancies mainly affecting elderly women $[1,2]$. Vulvar cancers predominantly metastasize via the lymphatic system, with the inguinofemoral lymph nodes a major site of metastases, and the extent of lymph node involvement is a strong predictor of disease recurrence and patient survival [3].

Regional lymph node metastases usually occur via the sentinel lymph node (SLN), which is the first draining lymph node to which the primary tumor is likely to spread [4].

Address for correspondence: Kazimierz Pityński, Department of Gynecology and Oncology, Jagiellonian University Medical College, Krakow, Poland E-mail: pitynski-k@wp.pl

Received: 14.11.2018; accepted: 25.03.2019; first published: 26.03.2019
The concept of sentinel node biopsy in vulvar cancer has been broadly studied and a consensus on its clinical value established, which maintains that women with unifocal primary vulvar cancer and tumour size $<4 \mathrm{~cm}$, without clinical evidence of inguinofemoral lymph node involvement, will benefit greatly from SLN sampling [4].

Historically, lymphatic vessels were considered passive participants in tumour metastasis, simply providing channels for tumour cells to transit to draining lymph nodes, and lymph nodes were likened to sieves, trapping cancer cells [5]. Currently, it is generally accepted that tumour-associated lymphatic vessels have active roles in cancer development, while lymph nodes are assumed to be a reservoir for tumour cells, from which they can take root and form metastatic tumours [6]. Tumour-induced lymphangiogenesis is believed to promote cancer metastasis to lymph nodes, although 
reported data are not equally robust for all types of tumours, and some contradictory results have been published [6]. The results of studies using animal models suggest that tumour-associated lymphangiogenesis has potential significance, not only at the primary site, but also in lymph nodes. Hence, it is likely that primary tumours can induce new lymphatic vessel growth in draining lymph nodes before metastasis [5, 7]. SLN lymphangiogenesis has been identified in cutaneous malignant melanoma, breast cancer, and extramammary Paget's disease; however, compared with tumour lymphangiogenesis, the role of lymph node lymphangiogenesis remains largely unexplored $[6,8,9]$.

The current study included women with unifocal squamous vulvar cancer and was designed to: (1) validate the feasibility of the SLN procedure for these patients, (2) estimate the incidence of skip metastases, and (3) evaluate the intensity of tumour and lymph node lymphangiogenesis in primary vulvar cancer, and determine the correlation between this process and tumour and lymph nodes using immunohistochemistry.

\section{MATERIALS AND METHOD}

This single centre prospective study was performed between 2010-2016 in the Department of Gynaecology and Oncology of the Jagiellonian University in Krakow, Poland, and was approved by the Jagiellonian University Ethics Board. Women with primary unifocal squamous vulvar cancer with tumours $<4 \mathrm{~cm}$, localized $>2 \mathrm{~cm}$ laterally from the median line, and with no evidence of inguinofemoral lymph node involvement, were considered eligible for the study. Patients with: (1) nonsquamous type vulvar cancer, (2) clinically or image positive inguinofemoral lymph nodes, (3) vulvar cancer recurrence, (4) history of vulvar/pelvic/abdominal radiation therapy, and (5) incomplete data, were excluded. After taking a history from each patient initially diagnosed with squamous vulvar cancer based on histopathological analysis of a vulvar biopsy, the patients were examined gynaecologically and rectally, the results reviewed, and additional laboratory and imaging tests performed. Subsequently, the patients considered by a gynecology/oncology consultant to be eligible for primary surgical treatment were included.

Surgical treatment and sentinel lymph node (SLN) detection procedure. Based on primary tumour diameter and localization, radical local excision or radical vulvectomy was performed, followed by unilateral systemic inguinofemoral lymphadenectomy. Patients with primary tumours localized $\leq 2 \mathrm{~cm}$ from the median line were excluded from the study. Prior to lymphadenectomy, SLN biopsies were performed in every patient by a high-volume radical vulvectomy surgeon, according to the trial protocol. For SLN detection, radiocolloid (Technetium-99 sulfur colloid) was injected intradermally $180( \pm 10)$ min prior to surgery, using a fourpoint injecting technique at 2, 5, 7, and 10 o'clock peritumourally. A gamma-probe was used for SLN identification before an inguinal incision was made to localize the optimal surgical approach. Isosulfan Blue $\left(1.0 \%, 1 \mathrm{~cm}^{3}\right.$ ) (Patent Blue V, Guerbert GmbH, Sulzbach, Germany) was also injected peritumourally using the same four-point injecting technique 20 $( \pm 5)$ min before surgery. The SLN procedure was performed prior to vulvectomy.
Histopathological evaluation and detection of lymphatic vessels. Postsurgical histopathological staging using FIGO 2009 classification and grading was performed on haematoxylin and eosin-stained slides, according to standard histopathological procedures, which confirmed the presence of squamous vulvar cancer in each patient [10]. Immunohistochemistry (IHC) of the lymphatic vessels detection was performed using a mouse monoclonal antibody against D2-40 (Anti-D240 antibody [D2-40]; Abcam, Cambridge, MA, USA), a new selective marker of lymphatic endothelium. IHC was performed on $4-5 \mu \mathrm{m}$ thick tissue sections. Slides were deparaffinized and dehydrated in $100 \%$ ethanol, washed in distilled water, and microwaved (600 W for $10 \mathrm{~min}$ and $5 \mathrm{~min}$ ) in antigen retrieval solution (EDTA, pH 8.0). Next, they were washed in distilled water, cooled at room temperature (RT) for 20-30 min, and immersed in $3 \% \mathrm{H}_{2} \mathrm{O}_{2}$ to block endogenous peroxidase. After washing in distilled water $(5 \mathrm{~min})$ and Wash Buffer (Tris-HCl; DakoCytomation, S3006; Agilent Technologies LDA, Ltd., Stockport, Cheshire, UK) twice for $5 \mathrm{~min}, 100 \mu \mathrm{l}$ of gammaglutamyltransferase 1 precursor antibody (GGT1; polyclonal (Thermo Scientific PA5-21344; Agiliens Technologies LDA, Ltd., Stockport, Cheshire, UK), diluted 1:400 in Dako Antibody Diluent with Background Reducing Components (Agiliens Technologies LDA, Ltd., Stockport,Cheshire, UK), was applied to each tissue section. Next, slides were incubated for $30 \mathrm{~min}$ at RT. After washing in Wash Buffer, secondary antibody (Dako Real EnVision HRP Rabbit/Mouse, K5007; Agiliens Technologies LDA, Ltd., Stockport, Cheshire, UK) was applied and incubated for $30 \mathrm{~min}$ at RT. peri-tumoural borders Visualization was performed using DAB (10-30 sec).

Lymphatic vessel density (LVD) using D2-40 staining, was evaluated in each tumour specimen, and in SLNs and non-SLNs, in 10 high-power fields (hpf; magnification x400), and expressed as the median number of lymphatic vessels per $1 \mathrm{~mm}^{2}$. In cases where more than one SLN was identified, the median LVD of all nodes was calculated and used for the analysis. Similarly, median LVD for non-SLNs was calculated and analyzed. Each sample was assessed blindly by two independent observers. In the case of any discrepancy between the observations, samples were examined again, together, to achieve a consensus.

Statistical analysis. The Shapiro-Wilk test confirmed that analyzed variables were non-normally distributed. Data are presented as medians and interquartile range (IQR) or number of cases and percentage. Mann-Whitney $U$ analysis was chosen for comparisons of independent groups of patients, while the Wilcoxon pair test was used to analyze LVD in consecutive lymph node samples. The KruskalWallis one-way analysis of variance with post-hoc testing when appropriate, was chosen to evaluate comparisons of more than two groups of variables. A univariate regression model was used to evaluate the relationship of LVD with clinicopathological characteristics. $\mathrm{p}=0.05$ was accepted as statistically significant. All calculations were performed using STATISTICA software v. 10 (StatSoft. 2011, Tulusa, OK, USA). 


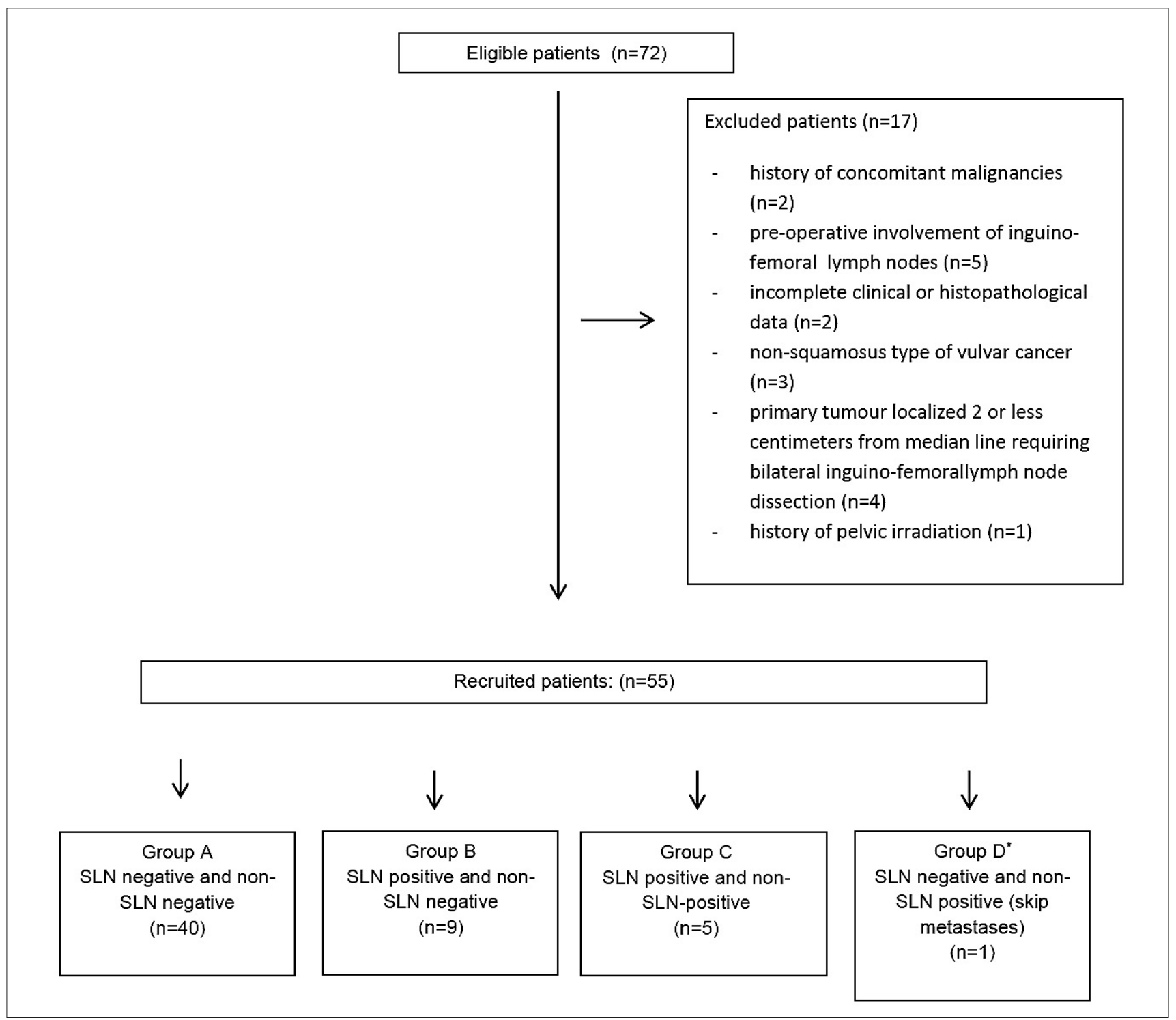

Figure 1. Flow diagram demonstrating participant recruitment procedure. *Group D was excluded from analyses due to the limited number of cases

\section{RESULTS}

Clinical characteristics. From a group of 72 participants who were prospectively enrolled into the study, 55 finally met the inclusion criteria (Fig. 1). Fourteen patients (25.45\%) underwent unilateral radical vulvectomy and 41 (74.55\%) underwent radical local tumour excision, followed in each case by unilateral systemic inguinofemoral lymphadenectomy. The median age of the included women was 72 years (IQR: 7.5 years). Detailed clinicopathological characteristics of patients are presented in Table 1.

Feasibility of the SLN procedure and risk of skip metastases. The SLN identification procedure using Isosulfan Blue failed in four women $(7.27 \%)$ and in one woman (1.82\%) using radiocolloid, indicating the high level of accuracy of each dye separately ( $92.73 \%$ and $98.18 \%$, respectively). Both methods of SLN detection achieved 100\% accuracy. Positive SLNs were identified in 14 (25.45\%) and positive non-SLNs were confirmed in five $(9.09 \%)$ patients, while $40(72.73 \%)$ participants were both SLN and non-SLN negative. Skip metastases, defined as positive non-SLN with negative SLN, were diagnosed in only one woman (1.82\%).

Based on lymph node involvement, the following four groups of patients were distinguished and included in further analysis: Group A $(\mathrm{n}=40)$, SLN negative and non-SLN negative; Group B $(n=9)$, SLN positive and non-SLN negative; Group C ( $n=5)$, SLN positive and non-SLN-positive; and Group D $(n=1)$, SLN negative and non-SLN positive (skip metastases). Group D was excluded from further evaluation due to the limited number of cases.

LVD in tumours and at the peri-tumour border. Intra and peri-tumour LVD was determined (Fig. 2). Both intra- and peri-tumour LVD were independent of patient age $(\mathrm{p}=0.672$ and 0.416 , respectively), tumour grade $(\mathrm{p}=0.881$ and 0.779 , respectively), disease stage ( $\mathrm{p}=0.956$ and 0.798 , respectively), and lymphatic spread ( $\mathrm{p}=0.649$ and 0.712 , respectively). Additionally, there were no differences in median intratumour or per-tumour LVD among Groups A, B, and C $(\mathrm{p}=0.273)$ (Tab. 2). However, peri-tumour median LVD was significantly higher compared with intra-tumour expression in Groups A, B, and C ( $<0.001)$ (Tab. 2), as well as in the 

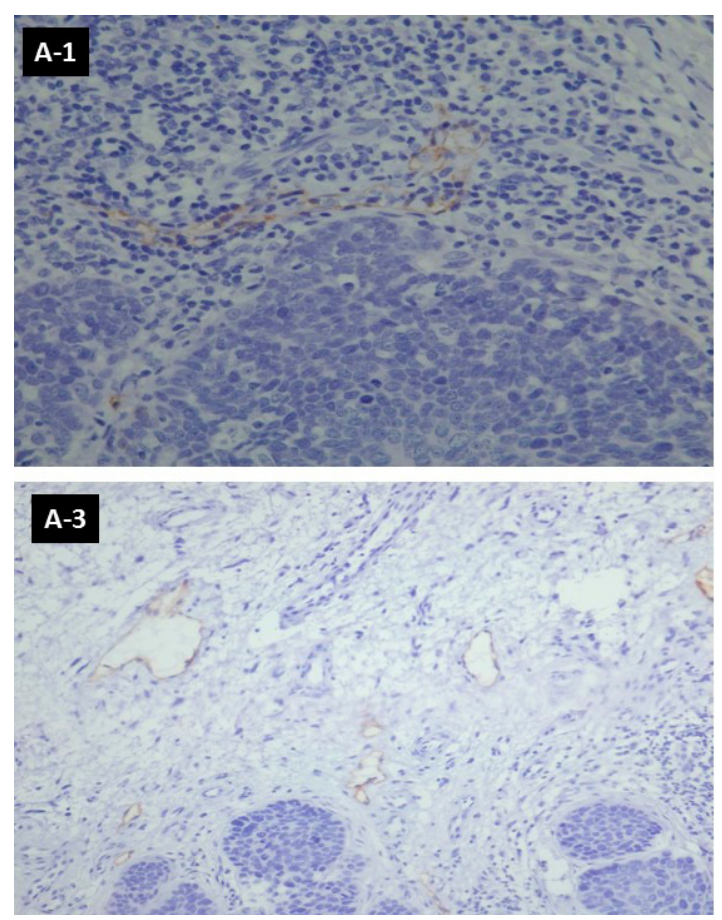
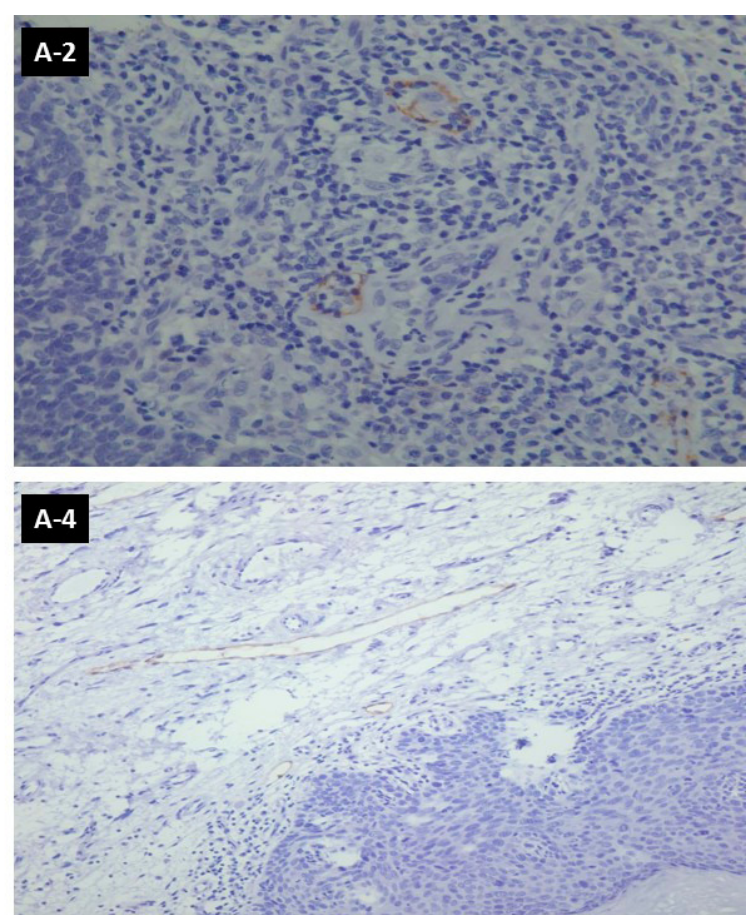

Figure 2. Lymphatic vessels in metastatic lymph nodes (1-A), non-metastatic lymph nodes (1-B), tumour specimen (1-C), tumour-stroma border (1-D), identified by immunochemistry staining using the D2-40 monoclonal mouse antibody (magnification X400)

entire patient cohort (1.753 IQR: 1.571 vs. 0.537 IQR:0.621); $\mathrm{p}<0.001)$.

LVD in sentinel and non-sentinel inguinal lymph nodes. LVD was confirmed in SLN and non-SLN (Fig. 2) and was independent from left/right localization $(p=0.254$ and 0.259 , respectively), patient age ( $\mathrm{p}=0.267$ and 0.501 , respectively), and tumour grade $(\mathrm{p}=0.143$ and 0.266 , respectively) in both groups; whereas, disease stage $(\mathrm{p}<0.001$ and $\mathrm{p}=0.003$, respectively) and lymphatic spread (both $\mathrm{p}<0.001$ ), were associated with LVD.

Median LVD in SLN was significantly lower in patients from Group A (negative SLN) than those in Groups B $(\mathrm{p}<0.001)$ and $\mathrm{C}(\mathrm{p}=0.005)$ (both positive SLN), while no significant differences in median LVD in SLN were observed between Groups B and C (Tab. 2). In non-SLN, the highest median LVD observed was in Group C (positive non-SLN), compared with Groups A ( $p=0.001)$ and $B(p=0.027)$ (both negative none-SLN), with no differences in median LVD expression observed between Groups A and B (Tab. 2).

Further detailed analysis revealed that in Group A (patients free from any nodular involvement), median LVD expression was higher in SLN, compared with matched non-SLN $(\mathrm{p}=0.012)$ (Tab. 2). In Group B, metastatic SLN exhibited significantly higher median LVD compared with matched involvement-free non-SLN ( $\mathrm{p}=0.015$ ) (Tab. 2). No differences in median LVD expression between SLN and matched nonSLN (both positive for metastases) were observed in Group C (Tab. 2).

\section{DISCUSSION}

The results of this study confirm the feasibility and clinical utility of SLN detection procedures in squamous vulvar cancer. The obtained findings are consistent with those of De Cicco et al. and Sliutz et al., who reported a $100 \%$ detection rate
Table 1. Clinical and pathological characteristics of patients with primary squamous vulvar cancer

\begin{tabular}{|c|c|}
\hline & Total cohort $(n=55)$ \\
\hline $\begin{array}{l}\text { Median age at therapy [years] }\left(\mathrm{IQR}^{*}\right) ; \\
\text { min.-max. }\end{array}$ & $\begin{array}{c}72(7.50) \\
64.00-87.00\end{array}$ \\
\hline $\begin{array}{c}\text { FIGO }^{* *} \text { stage } \\
\mathrm{I}_{\mathrm{A}} \\
\mathrm{I}_{\mathrm{B}} \\
\mathrm{II} \\
\mathrm{III} \\
\mathrm{III} \\
\mathrm{III} \\
\mathrm{IV} \\
\mathrm{C}\end{array}$ & $\begin{array}{c}0(0.00 \%) \\
40(72.73 \%) \\
0(0.00 \%) \\
10(18.18 \%) \\
4(7.27 \%) \\
1(1.82 \% \\
0(0.00 \%)\end{array}$ \\
\hline $\begin{array}{c}\text { Grade } \\
1 \\
2 \\
3\end{array}$ & $\begin{array}{c}31(56.36 \%) \\
21(38.19 \%) \\
3(5.45 \%)\end{array}$ \\
\hline $\begin{array}{l}\text { Adjuvant treatment } \\
\text { None } \\
\text { Radiotherapy (RTH) } \\
\text { Chemotherapy (CHT) }\end{array}$ & $\begin{array}{c}40(72.73 \%) \\
15(27.27 \%) \\
0(0.00 \%)\end{array}$ \\
\hline $\begin{array}{l}\text { History of CIN \# } \\
\text { Positive } \\
\text { Negative }\end{array}$ & $\begin{array}{l}12(21.82 \%) \\
43(78.18 \%)\end{array}$ \\
\hline $\begin{array}{c}\text { History of tobacco use } \\
\text { Yes } \\
\text { No }\end{array}$ & $\begin{array}{l}36(65.45 \%) \\
19(34.55 \%)\end{array}$ \\
\hline $\begin{array}{l}\text { History of vulvar lichen planus } \\
\text { Yes } \\
\text { No }\end{array}$ & $\begin{array}{l}1(1.82 \%) \\
54(98.18)\end{array}$ \\
\hline $\begin{array}{c}\text { History of vulvar lichen sclerosus } \\
\text { Yes } \\
\text { No }\end{array}$ & $\begin{array}{c}2(3.64 \%) \\
53(96.36 \%)\end{array}$ \\
\hline $\begin{array}{l}\text { Median number of SLN\#\# } \\
\left(I Q R^{*}\right) ; \text { min.-max. }\end{array}$ & $1(1)$ \\
\hline $\begin{array}{l}\text { Median number of non-SLN"\#\# } \\
\qquad\left(\mathrm{IQR}^{*}\right)\end{array}$ & $4(1)$ \\
\hline
\end{tabular}

"IQR - interquartile range; “" International Federation of Gynecology and Obstetrics classification of vulvar cancer; ${ }^{\#} \mathrm{CIN}$ - cervical intraepithelial neoplasia; ${ }^{\# \prime} \mathrm{SLN}$ - sentinel inguinofemoral lymph nodes; ${ }^{* \# \#}$ non-SLN - non-sentinel inguinofemoral lymph nodes. 
using Technetium-99-m-labeled nanocolloid, with or without blue dye $[11,12]$. Additionally a meta-analysis performed by Brincat et al. confirmed the safety and feasibility of SLN biopsy in vulvar cancer [13].

There is increasing evidence to support the promotion of metastasis by primary tumours through induction of the formation of a supportive microenvironment in a secondary organ site, termed the pre-metastatic niche [14]. Niche formation occurs as an ordered temporal sequence of events, one of which is lymphangiogenesis. There is experimental data demonstrating that primary tumours induce the growth of new lymphatic vessels within draining lymph nodes before they metastasize [15]. The expansion of the lymphatic network in draining lymph nodes has been referred to as the 'lymphvascular niche' [7]. It is likely that the endothelium of newly-formed lymphatic vessels promotes the lymphatic spread of tumours; however, the roles of lymphatic vessels within the pre-metastatic niche in the metastasis of human cancer are poorly understood and rarely investigated. Modification of the microenvironment to facilitate development of a secondary tumour in lymph nodes initiated by a primary tumour before, and independently of, the physical presence of metastatic cancer cells, has been observed in human cancers, including breast and tongue carcinomas [9]. The current study examined the process of lymphangiogenesis in squamous cell primary vulvar cancer by measuring lymphvascular density within tumours, peri-tumourally, and in sentinel and non-sentinel inguinal lymph nodes. Although LVD is not universally recognized as a measure of lymphangiogenesis, there is agreement that the lymphatic system initially present within and around a tumour generally responds to lymphangiogenic growth factors by proliferation and/or sprouting, and the density of lymphatic microvessels is significantly increased during tumour development, which indirectly indicates lymphangiogenesis supporting metastasis [16].

Lymph vascular density was higher in peritumour stroma than within tumours of the vulva, and both within- and peritumour LVD was independent of tumour stage. The difference in density of lymph vessels between the tumour and peritumour stroma is typical of both human tumours and animal models [17]. Although the intratumour lymphatic system provides an interface for lymphatic vessel invasion and metastasis, they are not considered crucial for increasing lymph node metastasis [18]. In some human cancers, intratumour lymphangiogenesis correlates with lymph node metastases, while in others there is no such correlation $[19,20$, 21]. The results of an investigation of vulvar cancer reported by Goes et al. suggest that increased intratumour LVD is associated with a lower rate of lymph node metastasis and favorable prognosis in vulvar squamous carcinomas [22]. Compared with tumour lymphangiogenesis, lymph node lymphangiogenesis is poorly investigated in human cancer, hence its role remains largely unexplored.

The most interesting finding of the current study is the demonstration for the first time of differences in lymphvascular density in inguinofemoral lymph nodes in squamous vulvar cancer. In cases with no lymph node metastases, the density of lymphatic vessels in the sentinel lymph nodes was higher than that in non-sentinel nodes. Median LVD was significantly lower in negative SLN, compared with both positive SLN and negative non-SLN. The data obtained in the current study confirm that the only independent factor significantly correlated with high LVD in SLN was the presence of metastases, whereas tumour stage, tumour grade, and patient age were not associated. Similar changes in lymph nodes were detected in animal models of cancer lymphangiogenesis [23]. The findings of Ishii et al. suggest that, in oral squamous cell carcinoma, the primary tumour actively induces lymphangiogenesis in SLNs prior to the onset of metastases, and that tumour-derived VEGF-C is important in this process [24]. Lymphangiogenesis in the regional lymph nodes is an independent prognostic marker in rectal cancer patients after neoadjuvant chemotherapy, as determined by Jacob et al. [25]. Increased SLN metastasis and lymphangiogenesis is associated with metastatic involvement of non-sentinel axillary lymph nodes in breast cancer [26].

The observations of squamous vulvar cancer in the current study appear to support the hypothesis that lymphangiogenesis and lymphatic remodeling are associated with the spread of lymphatic cancer. According to this hypothesis, primary tumours might prepare subsequent sites for metastasis. Hence, alterations in the lymphatic system of the tumour would facilitate the migration of tumour cells into sentinel lymph nodes [27]. New lymphatic vessels in tumour-draining lymph nodes could represent a metastatic niche for cancer cells, which facilitate further metastatic transformation of tumour cells, and potentially increase the spread of tumour cells to distant sites in the body [28]. Moreover, SLN lymphangiogenesis can contribute to the inhibition of anti-tumour-specific immune responses [29].

The large disproportion in the number of patients between the study groups may be considered a potential limitation of the study. Although the authors acknowledge that the results must be interpreted with caution; however, vulvar cancer is a rare gynaecological malignancy. Additionally, suspicion of lymph node involvement is a core contraindication for SLN procedure; therefore, in every patient lymph node metastases were mandatory excluded, based on clinical examination and imaging tests. This is the reason for the large disproportion between study groups, this is the result of the proper selection patients not influencing a collection bias. The strength of this study was its comprehensive analysis of LVD in SLN and nonSLN; additionally, every cases of vulvar cancer was confirmed in histopathological reevaluation of specimens that were independently assessed by two experienced pathologists to confirm their diagnoses and classifications.

Further studies are required to ascertain tumour-induced changes in regional and distant lymphatic systems, and their roles in cancer development and progression in the context of rapidly expanding targeted therapy approaches.

\section{CONCLUSIONS}

The data obtained allow the authors to make the following conclusions:

1) SLN detection by an experienced surgeon is a safe and feasible procedure in vulvar cancer, that can facilitate reduction of the adverse effects associated with inguinofemoral lymphadenectomy in selected cases.

2) A significantly higher median peri-tumour LVD was observed, compared with intra-tumour LVD, irrespective of tumour grade or disease stage, which was not associated with SLN or non-SLN involvement. 
3) Both SLN and non-SLNs showed significantly elevated median LVD values if they contained metastases.

4) In patients without nodular involvement, SLN showed significantly higher median LVD, compared with nonSLNs, which indicates SLN preparation for hosting metastases, and thus requires further investigation. Additionally, women with positive SLNs and non-SLNs did not show significant differences in median LVD between SLNs and non-SLNs.

5) Significantly higher median LVD expression in positive SLNs, compared with negative non-SLNs, was observed in women in Group B, which may be the result of SLNs involvement; however, the processes involved in preparing the lymph node for hosting metastases may also be important for generating LVD and cannot be ruled out.

\section{REFERENCES}

1. Banas T, Pitynski K, Jach R, Knafel A, Ludwin A, Juszczyk G, Nieweglowska D. Primary vulvo-vaginal cancers: Trends in incidence and mortality in Poland (1999-2012). Gynecol Obstet Invest. 2015; 80: 240-245.

2. Maclean AB, Jones RW, Scurry J, Neill S. Vulvar cancer and the need for awareness of precursor lesions. J Low Genit Tract Dis. 2009; 13: 115-117.

3. Covens A, Vella ET, Kennedy EB, Reade CJ, Jimenez W, Le T. Sentinel lymph node biopsy in vulvar cancer: Systematic review, metaanalysis, and guideline recommendations. Gynecol Oncol. 2015; 137: 351-361.

4. Hacker NF, Barlow EL. Sentinel node biopsy in vulvar cancer: A critical appraisal. Asian J Oncol. 2017; 3(1): 5-11.

5. Stacker SA, Williams SP, Karnezis T, Shayan R, Fox SB, Achen MG. Lymphangiogenesis and lymphatic vessel remodelling in cancer. Nat Rev Cancer. 2014; 14: 159-172.

6. Rahmani M, Mohammed S. Breast cancer metastasis and the lymphatic system. Oncol Lett. 2015; 10: 1233-1239.

7. Dieterich LC, Detmar M. Tumour lymphangiogenesis and new drug development. Adv Drug Deliv Rev. 2016; 99: 148-160.

8. Dadras SS, Lange-Asschenfeldt B, Velasco P, Nguyen L, Vora A, Muzikansky A, Jahnke K, Hauschild A, Hirakawa S, Mihm MC Detmar M. Tumour lymphangiogenesis predicts melanoma metastasis to sentinel lymph nodes. Mod Pathol. 2005; 18: 1232-1242.

9. Van den Eynden GG, Van der Auwera I, Van Laere SJ, Huygelen V, Colpaert CG, van Dam P, Dirix LY, Vermeulen PB, Van Marck EA. Induction of lymphangiogenesis in and around axillary lymph node metastases of patients with breast cancer. Br J Cancer. 2006; 95: 1362 1366.

10. NCCN Clinical Practice Guidelines in Oncology. Uterine Neoplasms. Version 1.2018 - October 13, 2017. Accessed from: https://www.nccn. $\mathrm{org} /$ professionals/physician_gls/pdf/uterine.pdf. on 2017-11-20.

11. De Cicco C, Sideri M, Bartolomei M, Grana C, Cremonesi M, Fiorenza M, Maggioni A, Bocciolone L, Mangioni C, Colombo N, Paganelli G. Sentinel node biopsy in early vulvar cancer. Br J Cancer. 2000; 82: 295-299.

12. Sliutz G, Reinthaller A, Lantzsch T, Mende T, Sinzinger H, Kainz C, Koelbl H. Lymphatic mapping of sentinel nodes in early vulvar cancer. Gynecol Oncol. 2002; 84: 449-452.
13. Brincat MR, Muscat Baron Y. Sentinel lymph node biopsy in the management of vulvar carcinoma: An evidence-based insight. Int J Gynecol Cancer. 2017; 27: 1769-1773.

14. Liu Y, Cao X. Characteristics and significance of the pre-metastatic niche. Cancer Cell 2016; 30: 669-681.

15. Hirakawa S, Kodama S, Kunstfeld R, Kajiya K, Brown LF, Detmar M. VEGF-A induces tumour and sentinel lymph node lymphangiogenesis and promotes lymphatic metastasis. J Exp Med. 2005; 201: 1089-1099.

16. Dadras SS, Paul T, Bertoncini J, Brown LF, Muzikansky A, Jackson DG, Ellwanger U, Garbe C, Mihm MC, Detmar M. Tumour lymphangiogenesis: a novel prognostic indicator for cutaneous melanoma metastasis and survival. Am J Pathol. 2003; 162: 1951-1960.

17. Li S, Li Q. Cancer stem cells, lymphangiogenesis, and lymphatic metastasis. Cancer Lett. 2015; 357: 438-447.

18. Ji R. Lymphatic endothelial cells, tumour lymphangiogenesis and metastasis: new insights into intratumoural and peritumoural lymphatics. Cancer Metastasis Rev. 2006; 25: 677-694.

19. Wong SY, Haack H, Crowley D, Barry M, Bronson RT, Hynes RO. Tumour-secreted vascular endothelial growth factor- $\mathrm{C}$ is necessary for prostate cancer lymphangiogenesis, but lymphangiogenesis is unnecessary for lymph node metastasis. Cancer Res. 2005; 65: 97899798.

20. Beasley NJ, Prevo R, Banerji S, Leek RD, Moore J, van Trappen P, Cox G, Harris AL, Jackson DG. Intratumoural lymphangiogenesis and lymph node metastasis in head and neck cancer. Cancer Res. 2002; 62: 1315-1320.

21. Maula SM, Luukkaa M, Grenman R, Jackson D, Jalkanen S, Ristamäki R. Intratumoural lymphatics are essential for the metastatic spread and prognosis in squamous cell carcinomas of the head and neck region. Cancer Res. 2003; 63: 1920-1926.

22. Goes RS, Carvalho JP, Almeida BG, Bacchi CE, Goes JC, Calil MA, Baracat EC, Carvalho FM. Intratumoural lymphatic vessel density in vulvar squamous cell carcinomas: A possible association with favorable prognosis. Int J Gynecol Pathol. 2012; 31:8-14.

23. Christiansen A, Detmar M. Lymphangiogenesis and Cancer. Genes Cancer. 2011; 2: 1146-1158.

24. Ishii K, Chikamatsu, Kakakura K, Miyata M, Furuya N, Masuyama K. Primary tumour induces sentinel lymph node lymphangiogenesis in oral squamous cell carcinoma. Oral Oncol. 2010; 46: 373-378.

25. Jakob C, Aust DE, Liebscher B, Baretton GB, Datta K, Muders MH. Lymphangiogenesis in regional lymph nodes is an independent prognostic marker in rectal cancer patients after neoadjuvant treatment. PLoS ONE. 2011; 611: e27402.

26. Van den Eynden GG, van den Berghe MK, van Dam PJ, Colpaert CG, van Dam P, Dirix LY, Vermeulen PB, Van Marck EA. Increased sentinel lymph node lymphangiogenesis is associated with nonsentinel axillary lymph node involvement in breast cancer patients with a positive sentinel node. Clin Cancer Res. 2007; 13(18): 5391-5397.

27. Wakisaka N, Hasegawa Y, Yoshimoto S, Miura K, Shiotani A, Yokoyama J, Sugasawa M, Moriyama-Kita M, Endo K, Yoshizaki T. Primary tumour-secreted lymphangiogenic factors induce pre-metastatic lymphvascular niche formation at sentinel lymph nodes in oral squamous cell carcinoma. PLoS ONE. 2015; 10: e0144056.

28. Kurahara H, Takao S, Shinchi H, Maemura K, Mataki Y, Sakoda M, Hayashi T, Kuwahata T, Minami K, Ueno S, Natsugoe S. Significance of lymphangiogenesis in primary tumour and draining lymph nodes during lymphatic metastasis of pancreatic head cancer. J Surg Oncol. 2010; 102: 809-815.

29. Cochran AJ, Huang RR, Lee J, Itakura E, Leong SP, Essner R. Tumourinduced immune modulation of sentinel lymph nodes. Nature Rev Immunol. 2006; 6: 659-670.

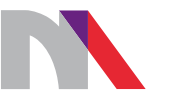

Ministry of Science and Higher Education

Republic of Poland

Generation of the DOI (Digital Object Identifier) - task financed under the agreement No. 618/P-DUN/2019 by the Minister of Science and Higher Education 